\title{
CAPACIDADES DINÂMICAS PARA AUXILIAR A TRANSFORMAÇÃO DIGITAL DAS ORGANIZAÇÕES INTENSIVAS DO CONHECIMENTO
}

\author{
Eliane Duarte Ferreira Ms. ${ }^{1}$; \\ Neri dos Santos Dr. ${ }^{2}$; \\ Patrícia de Sá Freire Dra. ${ }^{3}$
}

\begin{abstract}
This article aims to identify how dynamic capabilities can assist organizations in the digital transformation process. This is a systematic review of the literature, based on the Scopus and WOS database, published in the last 05 years using the descriptors Dynamic capabilities OR absorptive capacity AND digital transformation. The investigation sought to identify case studies with empirical evidence on the researched topic. The main results point out that dynamic capabilities can support organizations in the process of digital transformation as they allow them to adapt to business ecosystems, to shape them through innovation and collaboration with other institutions and in the organizational transformation itself.
\end{abstract}

Keywords: digital transformation; knowledge managemen; dynamic capabilities.

\section{RESUMO}

Este artigo tem como objetivo identificar como as capacidades dinâmicas podem auxiliar as organizações no processo de transformação digital. Trata-se de uma revisão sistemática da literatura, a partir da base de dados Scopus e WOS, publicadas nos últimos 05 anos utilizando os descritores Dynamic capabilities OR absorptive capacity AND digital transformation. A investigação buscou identificar estudos de caso com evidências empíricas sobre o tema pesquisado. Os principais resultados apontam que as capacidades dinâmicas podem apoiar as organizações em processo de transformação digital uma vez que permitem adaptação aos ecossistemas de negócios, moldá-los por intermédio da inovação e da colaboração com outras instituições e na própria transformação organizacional.

Palavras chave: transformação digital; gestão do conhecimento; capacidades dinâmicas.

\footnotetext{
${ }^{1}$ Programa de Pós-Graduação em Engenharia e Gestão do Conhecimento - Universidade Federal de Santa Catarina (UFSC) Florianópolis - Brasil. E-mail: elianedfer@gmail.com
${ }^{2}$ Programa de Pós-Graduação em Engenharia e Gestão do Conhecimento - Universidade Federal de Santa Catarina (UFSC) Florianópolis - Brasil. E-mail: nerisantos@ gmail.com
3 Programa de Pós-Graduação em Engenharia e Gestão do Conhecimento - Universidade Federal de Santa Catarina (UFSC) Florianópolis - Brasil. E-mail:patriciadesafreire@ gmail.com




\section{INTRODUÇÃO}

O tema Capacidades dinâmicas (CD) ou Dynamic Capabilities tem despertado o interesse tanto no meio corporativo como no meio acadêmico. Isso porque tem ficado cada vez mais evidente para os gestores de organizações que compreender e identificar as capacidades dinamicas organizacionais conduz a uma gestão mais forte e competitiva.

Em ecossistemas organizacionais voláteis (Bhamra; Dani \& Burnard, 2011), as vantagens competitivas são temporárias e as organizações enfrentam desafios para enfrentar dinamicamente essas mudanças externas e turbulências.

Teece (2016) aponta que estudos sobre (CDs) vem sendo vistas como acontecimentos organizações capazes de auxiliar no desafio de manter organizações vivas e competitivas no mercado, já que as empresas são mais bem-sucedidas quando desenvolvem habilidades e competências que lhes permitem ganhar manter vantagem competitiva em ambiente em mudança (Helfat \& Winter, 2011; Surmeier, 2020).

A digitalização abriu uma ampla gama de possibilidades para as empresas interagirem com os clientes, o que levou a inovações de modelos de novos negócios e inesperados. Para sobreviver nesse ambiente ágil e volátil é fundamental entender com precisão como as organizações gerenciam sua transformação para capitalizar os benefícios das tecnologias digitais (HESS, MATT, BENLIAN \& WIESBÖCK, 2016).

A transformação digital surgiu como um termo que descreve partida dos esforços de digitalização para uma revisão completa da políticas, processos e serviços imprescindíveis para as organizações sobreviverem em um ecossistema dinâmico.

A convergência desses dois temas vem despertando interesse no meio acadêmico, bem como no ambiente corporativo, nos seus mais diversos segmentos, que vão desde a área estratégica, passando pela gestão de recursos humanos, marketing, inovação, empreendedorismo e gestão da informação e do conhecimento.

Assim, considerando a relevância do tema, este trabalho tem como objetivo identificar como as capacidades dinâmicas podem apoiar as organizações no processo de transformação digital, por meio de uma revisão sistemática da literatura, nas bases de dados Scopus e Web of Science, nos períodos de 2015 a 2019. Assim sendo, a pesquisa procura responder a seguinte 
questão de pesquisa: Como as capacidades dinâmicas podem apoiar as organizações no processo de transformação digital?

Este artigo está estruturado em cinco seções: a primeira e presente seção corresponde à introdução. A segunda apresenta os principais conceitos relacionados à CD e Transformação digital. A terceira seção apresenta o método utilizado no levantamento dos dados. A quarta seção constitui a análise de como as capacidades dinâmicas podem auxiliar as organizações no processo de transformação digital identificados na literatura e a quinta seção contempla as considerações finais.

\section{FUNDAMENTAÇÃO TEÓRICA}

$\mathrm{Na}$ sequência, apresenta-se os fundamentos teóricos relacionados aos temas "transformação digital" e "capacidades dinâmicas", com o intuito apenas de conceituá-los, conforme abordados nesta pesquisa.

\subsection{TRANSFORMAÇÃO DIGITAL}

A nova era digital tem conduzidos as organizações a se moldarem e se adaptarem a essa nova realidade para permanecerem competitivas no mercado. A criação de conteúdo, aplicativos, ferramentas e serviços digitais através do uso das TICs estão sendo cada vez mais utilizadas para sustentar vantagem competitiva e os recursos digitais passam a fazer parte da dimensão dos recursos organizacionais vitais para a sobrevivência do negócio.

Warner \& Wäger (2019) salientam que as organizações precisam estar preparadas para a transformação digital que difere das formas tradicionais de mudança estratégica, na medida que as tecnologias digitais aceleraram a velocidade da mudança, resultando em muito mais volatilidade ambiental, complexidade e incerteza.

Dugstad, Eide, Nilsen, \& Eide (2019) afirmam que a transformação digital surgiu como um termo que descreve o ponto de partida dos esforços de digitalização para uma revisão 
completa das políticas, processos e serviços, a fim de criar uma experiência de usuário mais simples, para os cidadãos e para os trabalhadores da linha da frente da produção.

Em função da contemporaneidade do tema, ainda existem poucas pesquisas teóricas ou empíricas que evidenciem como as organizações são transformadas digitalmente.

Entretanto, vários autores (Brynjolfsson \& Hitt, 2000; Liu, Chen, \& Chou, 2011; Fitzgerald, Kruschwitz, Bonnet, \& Welch, 2014; Hess, Benlian, \& Wiesböck, 2016; Rogers, 2016; Santos, 2019) têm procurado definir o termo "transformação digital”, a luz da realidade atual. A Tabela 1 apresenta os construtos propostos por esses autores.

Tabela 1 - Construtos transformação digital

\begin{tabular}{ll}
\hline \hline Autor & Construto de transformação digital \\
\hline \hline Brynjolfsson \& Hitt (2000) & $\begin{array}{l}\text { Estruturar novas operações de negócios facilitar e } \\
\text { alavancar plenamente a competência central das } \\
\text { empresas por meio da tecnologia digital para obter } \\
\text { vantagem competitiva. }\end{array}$ \\
\hline \hline Fitzgerald, Kruschwitz, Bonnet, \& Welch (2014) & $\begin{array}{l}\text { O uso de novas tecnologias digitais (mídias sociais, } \\
\text { dispositivos móveis, análises ou dispositivos } \\
\text { incorporados) para permitir grandes melhorias nos } \\
\text { negócios, como melhorar a experiência do cliente, } \\
\text { otimizar operações ou criar novos modelos de } \\
\text { negócios. }\end{array}$ \\
\hline \hline Chen, \& Chou, (2011) & $\begin{array}{l}\text { Transformação organizacional que integra tecnologias } \\
\text { digitais e processos de negócios em uma economia } \\
\text { digital. }\end{array}$ \\
\hline \hline Rogers, D. L. (2016) & $\begin{array}{l}\text { A transformação digital não é fundamentalmente } \\
\text { sobre tecnologia, mas sobre estratégia. }\end{array}$ \\
\hline \hline Hess, Benlian, \& Wiesböck, (2016) & $\begin{array}{l}\text { A transformação digital está preocupada com as } \\
\text { mudanças que as tecnologias digitais podem trazer no } \\
\text { modelo de negócios de uma empresa, que resultam em } \\
\text { produtos alterados ou estruturas organizacionais ou na } \\
\text { automação de processos. }\end{array}$ \\
\hline \hline Santos, N. (2019) & $\begin{array}{l}\text { Transformação digital é a mudança fundamental das } \\
\text { economias, organizaçóes e sociedades, causadas pela } \\
\text { aplicação abrangente de tecnologias digitais e modelos } \\
\text { de negócios digitais disruptivos. }\end{array}$ \\
\hline \hline
\end{tabular}

Fonte: Dados da pesquisa (2019)

Warner \& Wäger (2019) chamam atenção para a diferenciação de transformação digital para outras formas de mudança estratégica isso porque tecnologias digitais aceleraram a velocidade da mudança, resultando em muito mais volatilidade ambiental, complexidade e incertezas. 
Porém, Vial (2019) ressalta que a tecnologia é apenas uma peça do quebra cabeça complexo que precisa ser resolvido para que as organizações continuem competitivas no mercado. Aspetos como estratégia, mudanças incluindo processos, cultura, análise de ambiente, devem fornecer a capacidade de gerar novos caminhos para a criação de valor, mas é inegável que a transformação digital com seus recursos e capacidades podem gerar a vantagem competitiva da empresa (BARNEY, 1991).

\subsection{CAPACIDADES DINÂMICAS}

Estudos sobre capacidades dinâmicas emergiram na década de 90, quando Teece, Pisano e Shuen iniciaram as primeiras pesquisas sobre o tema, definindo capacidade dinâmica como a capacidade da empresa de integrar, construir e configurar recursos internos e competências externas para lidar com mudanças rápidas do ambiente (TEECE \& PISANO, 1994; TEECE, PISANO \& SHUEN, 1997).

Uma década mais tarde, Teece, (2007, pág. 1319) complementa essa definição e classifica capacidade dinâmica em uma dimensão "intensamente empreendedora", isso porque além da necessidade das organizações adaptar-se aos ecossistemas de negócios precisam também moldá-los por meio de inovação e da colaboração com outras instituições e chama atenção para três capacidades fundamentais:

a) Sensing - Capacidade de detectar e moldar ameaças e oportunidades;

b) Seizing - Capacidade de aproveitar as oportunidades;

c) Transforming - Capacidade de manter a competitividade.

A capacidades de detectar e moldar ameaças e oportunidades são consideradas atividades sensoriais de detecção, envolvem a identificação de oportunidade nos ambientes internos e externos. A capacidade de aproveitar oportunidades diz respeito a criar e combinar novos modelos de negócios a fim de explorá-los. A capacidade de manter competitividade refere-se à transformação da empresa necessária para explorar essas novas oportunidades.

Mendonca \& de Andrade (2018) visualizam capacidades dinâmicas como o agrupamento de habilidades, comportamentos e capacidades organizacionais, assim como 
processos e rotinas, que levam a empresa a se diferenciar no mercado competitivo frente aos seus concorrentes.

Dabab \& Weber (2018) enfatizam que os usos das capacidades dinâmicas tendem a explicar o desempenho superior no nível da empresa a longo prazo, em ambientes tecnológicos dinâmicos e de clientes que mudam rapidamente movidos ou pela inovação e pela abertura global. O poder da capacidade dinâmica de uma organização é o elemento que determina a velocidade, o alinhamento da locação de recursos da empresa, levando em conta

Warner \& Wäger (2019) destacam que a estrutura de recursos dinâmicas fornece uma visão poderosa para a compreensão de mudanças estratégicas organizacionais (Teece, 2016) uma vez que são baseados em inovação e é isso que distingue capacidades dinâmicas de recursos operacionais de uma empresa, conforme demonstrado na Tabela 2. Observa-se que as capacidades operacionais são comuns no sentido em que ajudam uma empresa no presente, mantendo o status quo, mas isso a deixa vulnerável às mudanças ambientais. As capacidades dinâmicas referem-se a práticas concretas de mudanças de processos e servem ao propósito de alterar recursos operacionais nas organizações (Konlechner, Müller, \& Güttel, 2018).

Tabela 2 - Diferenças de capacidades comuns e capacidades dinâmicas

\begin{tabular}{|c|c|c|}
\hline & Capacidades Comuns & Capacidade Dinâmicas \\
\hline Finalidade & $\begin{array}{l}\text { Eficiência técnica nos negócios, } \\
\text { funções }\end{array}$ & $\begin{array}{l}\text { Congruência com as } \\
\text { necessidades do cliente e com as } \\
\text { oportunidades tecnológicas e de } \\
\text { negócios }\end{array}$ \\
\hline Esquema Tripartido & "Operar, administrar, e governar & Sentir, aproveitar e transformar \\
\hline Rotinas principais & Melhores práticas & Processos (atualizados) \\
\hline ÊEfase gerencial & Controle de custo & \begin{tabular}{llr} 
Orquestração, & \multicolumn{2}{l}{ liderança e } \\
aprendizado \\
empresariais
\end{tabular} \\
\hline Prioridade & Fazendo as coisas certas & $\begin{array}{l}\text { Fazendo as coisas certas no } \\
\text { momento certo }\end{array}$ \\
\hline Imitabilidade & "Relativamente imitável & Inimitável \\
\hline Resultado & $\begin{array}{l}\text { Aptidão técnica (eficiência } \\
\text { estática) }\end{array}$ & $\begin{array}{l}\text { Aptidão evolutiva (em } \\
\text { andamento, aprimoramento de } \\
\text { capacidade e alinhamento }\end{array}$ \\
\hline
\end{tabular}

Fonte: Adaptado de Teece (2014)

Essas capacidades dinâmicas incluem a detecção, apreensão e transformação necessárias para projetar e implementar um modelo de negócios. Eles podem permitir que uma 
empresa atualize seus recursos comuns e direcione-os, e os recursos dos parceiros, a empreendimentos de alto retorno. Isso requer o "desenvolvimento e a coordenação, ou orquestração", dos recursos da empresa (e das empresas parceiras) para tratar e até moldar as mudanças no mercado ou no ambiente de negócios em geral (TEECE, 2018).

\section{PROCEDIMENTOS METODOLÓGICOS}

O método de revisão sistemática de literatura refere-se à aplicação de estratégias científicas que limitem a seleção de artigos que sintetizem estudos pertinentes e relevantes a um tema específico (Torres-Carrión, González-González, Aciar, \& Rodríguez-Morales, 2018) Esse tipo de estudo se propõe a sintetizar e analisar o conhecimento cientifico já publicado utilizando uma metodologia passível de ser avaliada e reproduzida

Baseado nessa concepção, esta revisão sistemática tem como objetivo geral identificar como as capacidades dinâmicas podem apoiar as organizações no processo de transformação digital e como objetivos específicos: (i) pesquisar estudos empíricos acerca do assunto; (ii) descrever os métodos de pesquisa utilizados e (iii) analisar os objetivos e os principais resultados obtidos.

A busca sistemática na literatura, aconteceu no dia 12 de agosto de 2019, nas bases de dados Scopus e Web of Science com os descritores "Dynamic capabilities" OR "absortive capacity" AND "digital transformation" em artigos indexados em periódicos e artigos publicados em anais de eventos e conferências nos últimos cinco anos. Quanto aos critérios de inclusão, procurou-se por estudos que disponibilizassem acesso ao texto completo nos idiomas português e inglês.

\section{APRESENTAÇÃO E ANÁLISE DOS RESULTADOS}

Foram encontrados 140 estudos de interesse na base Scopus e 74 na WOS. Esses artigos foram exportados para o Mendeley, onde foi verificada a duplicação de materiais e a disponibilidade de acesso. A Tabela 3 apresenta esses resultados. 
Tabela 3 - Resultado da busca por palavra-chave

\begin{tabular}{crr}
\hline Palavras-chave & Frequência Scopus & Frequência WOS \\
\hline $\begin{array}{c}\text { Dynamic capabilities } \boldsymbol{O R} \\
\text { absortive capacity }\end{array}$ & 3.840 & 10,717 \\
Digital transformation & & 11.919 \\
$\begin{array}{c}\text { Dynamic capabilities And } \\
\text { Digital transformation }\end{array}$ & 140 & 74 \\
\hline
\end{tabular}

Fonte: Dados da pesquisa (2019)

A seleção dos artigos foi realizada, em duas etapas:

Na primeira etapa, as buscas aconteceram por intermédio da leitura dos descritores em títulos, resumos e palavras chave o que resultou numa coleção de 40 artigos;

Na segunda etapa, foram aplicados os critérios de exclusão, descartando-se artigos que não tivessem com foco organizações do conhecimento, o que resultou 14 artigos, sendo 7 destes estudos de caso.

A síntese dos resultados obtidos pode ser observada na Tabela 4 a seguir:

Tabela 4 - Resultados

\begin{tabular}{cccr}
\hline Base & Recuperados & Pré-selecionados & Selecionados \\
\hline Scopus & 21 & 20 & 9 \\
Web of Science & 19 & 8 & 5 \\
\hline Total & 40 & 28 & 14
\end{tabular}

Fonte: Dados da pesquisa (2019)

O Quadro 1 apresenta-se a descrição de autoria, ano e título de publicação dos 14 artigos selecionados.

Quadro 1 - Publicações selecionadas para análise

\begin{tabular}{|c|l|l|}
\hline Id & Autoria/Ano & Título \\
\hline 1 & Warner \& Wäger (2019) & $\begin{array}{l}\text { Building dynamic capabilities for digital } \\
\text { transformation: An ongoing process of strategic } \\
\text { renewal }\end{array}$ \\
\hline 2 & Vial (2019) & $\begin{array}{l}\text { Understanding digital transformation: A review and a } \\
\text { research agenda }\end{array}$ \\
\hline 3 & Riera \& Iijima (2019) & $\begin{array}{l}\text { The Role of IT and Organizational Capabilities on Digital } \\
\text { Business Value }\end{array}$ \\
\hline 4 & Sánchez \& Zuntini (2018) & $\begin{array}{l}\text { Organizational readiness for the digital transformation: } \text { a } \\
\text { case study research }\end{array}$ \\
\hline 5 & Dabab \& Weber (2018) & $\begin{array}{l}\text { Business Intelligence and Data Analytics as a Driver of } \\
\text { Dynamic Capability Strategic Approach }\end{array}$ \\
\hline
\end{tabular}




\begin{tabular}{|c|c|c|}
\hline 6 & $\begin{array}{l}\text { Konlechner, Müller, \& Güttel. } \\
\text { (2018) }\end{array}$ & $\begin{array}{l}\text { A dynamic capabilities perspective on managing } \\
\text { technological change: a review, framework and research } \\
\text { agenda }\end{array}$ \\
\hline 7 & $\begin{array}{l}\text { De Mendonca \& De Andrade } \\
\text { (2018) }\end{array}$ & $\begin{array}{l}\text { Use of the elements of digital transformation in dynamic } \\
\text { capabilities in a Brazilian capital [Uso de Elementos da } \\
\text { Transformação Digital nas Capacidades Dinămicas em } \\
\text { uma Capital Brasileira] }\end{array}$ \\
\hline 8 & $\begin{array}{l}\text { De Mendonca \& De Andrade } \\
\text { (2018) }\end{array}$ & $\begin{array}{l}\text { Microfoundations of dynamic capabilities and their } \\
\text { relations with elements of digital transformation in } \\
\text { Portugal }\end{array}$ \\
\hline 9 & Hsu, Tsaih, \& Yen (2018) & $\begin{array}{l}\text { The Evolving Role of IT Departments in Digital } \\
\text { Transformation }\end{array}$ \\
\hline 10 & Sanchez (2017) & $\begin{array}{l}\text { A framework to assess organizational readiness for the } \\
\text { digital transformation }\end{array}$ \\
\hline 12 & Korhonen \& Halen (2017) & Enterprise Architecture for Digital Transformation \\
\hline 11 & $\begin{array}{l}\text { Carcary, Doherty, \& Conway } \\
\text { (2016) }\end{array}$ & $\begin{array}{l}\text { A dynamic capability approach to Digital } \\
\text { transformation: A focus on key foundational themes }\end{array}$ \\
\hline 13 & Karimi \& Walter (2015) & $\begin{array}{l}\text { The Role of Dynamic Capabilities in Responding to Digital } \\
\text { Disruption: A Factor-Based Study of the Newspaper } \\
\text { Industry }\end{array}$ \\
\hline 14 & Liu, Chen, \& Chou (2011) & $\begin{array}{l}\text { Resource fit in digital transformation Lessons learned } \\
\text { from the CBC Bank } \\
\text { global e-banking project }\end{array}$ \\
\hline
\end{tabular}

Fonte: Dados da pesquisa (2019)

Dentre os 14 artigos listados, foram selecionados 7 estudos de caso a serem analisados no contexto desta pesquisa conforme apresentado no Quadro 2. A seleção dos artigos levou em consideração os objetivos propostos nesta pesquisa.

Quadro 2 - Referências Selecionadas

\begin{tabular}{|l|l|c|c|l|l|}
\hline Id & Autoria & Abordagem & Técnica & Público e amostra & País \\
\hline 1 & $\begin{array}{l}\text { Warner \& Wäger } \\
(2019)\end{array}$ & Qualitativa & $\begin{array}{l}\text { Observação, entrevistas } \\
\text { semiestruturadas e } \\
\text { análise de documentos }\end{array}$ & $\begin{array}{l}\text { 4 empresas estabelecidas na } \\
\text { Alemanha (18 entrevistados) }\end{array}$ & Alemanha \\
\hline 3 & $\begin{array}{l}\text { Sánchez \& } \\
\text { Zuntini (2018) } \\
\text { semiestruturadas }\end{array}$ & $\begin{array}{l}\text { Qualitativa } \\
\text { médio porte. (Entrevista com } \\
\text { proprietários e gerentes. }\end{array}$ & $\begin{array}{l}\text { Argentina } \\
\text { 100 PMEs japonesas. } \\
\text { (34vrespostas) }\end{array}$ & Japão \\
\hline 4 & $\begin{array}{l}\text { Riera \& Iijima } \\
(2019)\end{array}$ & Quantitativa & Questionários \\
8 & $\begin{array}{l}\text { De Mendonca \& } \\
\text { De Andrade }\end{array}$ & Quantitativa & Questionários & $\begin{array}{l}\text { Empresários e gestores de TI. } \\
\text { Enviados 247 formulários com } \\
137 \text { válidos }\end{array}$ & Portugal \\
\hline 9 & $\begin{array}{l}\text { Sánchez (2017) } \\
\text { (2018) }\end{array}$ & Qualitativa & Questionários & $\begin{array}{l}\text { Gerentes de negócios e de TI } \\
(\text { 53 questionários válidos) }\end{array}$ & Brasil \\
\hline 11 & $\begin{array}{l}\text { Hsu, Tsaih \& Yen } \\
(2018)\end{array}$ & Qualitativa & $\begin{array}{l}\text { Euncionários do Museu do } \\
\text { Palácio Nacional (NPM) }\end{array}$ & Taiwan \\
\hline 14 & $\begin{array}{l}\text { Liu, Chen, \& } \\
\text { Chou (2011) }\end{array}$ & Qualitativa & $\begin{array}{l}\text { Entrevista em } \\
\text { profundidade }\end{array}$ & $\begin{array}{l}\text { Funcionários do Banco } \\
\text { Mundial da CBC 17 } \\
\text { entrevistas }\end{array}$ & Taiwan \\
\hline
\end{tabular}

Fonte: Dados da pesquisa (2019) 
O estudo de caso foi realizado por Warner \& Wäger (2019) e explora como as empresas tradicionais da indústria na Alemanha constroem capacidades dinâmicas para a transformação digital. Nessa pesquisa os autores entrevistaram 17 pessoas de 4 empresas e com bases nesses resultados relatam que transformação digital tem seu ponta pé com a renovação estratégica contínua no modelo de negócios. Mostram que a melhoria da maturidade digital da força de trabalho é uma capacidade dinâmica fundamental para essas transformações. Ressaltam que essas descobertas têm ressonância com pesquisas sobre inovações de gestão, como a construção de novas capacidades de governança digital para transformar digitalmente as abordagens colaborativas internas e enfatizam a importância de as organizações navegarem na inovação em ecossistemas, como uma forma emergente de inovação de modelo de negócio radical.

A pesquisa de Sánchez (2018), apresenta como objetivo formular uma estrutura que aborde recursos, capacidades dinâmicas e opções de gestão necessárias para responder ao novo ambiente. Segundo o entendimento das autoras as capacidades dinâmicas são as capacidades da empresa de construir, integrar e reconfigurar competências internas e externas para abordar os ambientes em rápida mutação (Teece, Pisano, \& Shuen, 1997). O estudo de caso apresenta 3 empresas de pequeno e médio porte da Argentina onde foram entrevistados seus respectivos gestores. Foram levantadas questões relativas a compreender o ambiente dessas organizações, quais barreiras encontradas para gerir o negócio e o que consideravam importantes para a geração de valor organizacional. Com base nesses resultados foi proposta uma estrutura baseada no Modelo das Cinco Forças (Porter \& Heppelmann 2014) com foco para: (i) absorção do impacto das inovações digitais nas forças externas; (ii) estratégias adequadas resultantes de uma análise da cadeia de valor e (iii) adequação do uso recursos e capacidades nas estratégias.

Em um segundo artigo, publicado somente por Sánchez (2017), discorre sobre uma pesquisa quantitativa realizada com 53 questionários respondidos por gerentes de negócios e de TI de uma empresa brasileira. O objetivo do artigo foi descrever o uso e a importância de elementos de transformação digital (IoT, Big Data e IA) como suporte a processos de capacidades dinâmicas em organizações de uma capital brasileira. Foi identificado que, na percepção dos gerentes de negócios e de TI, os elementos de transformação digital, mesmo com informações relativamente baixas, principalmente de IoT e IA, com foco maior em Big Data, são avaliados como importantes, tanto hoje quanto, na prática, até 2025 , em processos envolvendo as capacidades dinâmicas de analisar o ambiente (Sensing), aproveitando 
(Gerenciando

Ameaças/Transformando). Com maior ênfase no Big Data e nos processos da capacidade dinâmica de aproveitar como oportunidades.

O estudo de caso foi realizado por Riera \& lijima (2019), o qual fornece insights práticos sobre como as Pequenas e Médias Empresas (PMEs) podem se preparar para adotar as tecnologias digitais. Este estudo estende a literatura da Transformação Digital, em particular, como as capacidades dinâmicas funcionam juntas para permitir o Digital Business Value. A população-alvo da pesquisa são 100 PMEs japonesas que foram premiadas pelo Ministério da Economia, Comércio e Indústria devido à sua utilização efetiva do desempenho de negócios de suporte de TI na lista de "Seleção Competitiva de PME de Estratégia de TI 100" nos anos 2015, 2016 e 2017. A coleta de dados foi realizada com questionários enviados a todas as organizações premiadas e coletadas 34 respostas. Como resultados foram evidenciados que recursos de TI, assim como outros recursos organizacionais vinculados a gestão de riscos, planejamento de negócios, disseminação interna do conhecimento e inovação conduzem a criação de valor e consequentemente são elementos alicerçam a organização no processo de transformação digital.

O estudo de caso foi de De Mendonça \& De Andrade (2018) identificam as relações entre elementos da transformação digital com os microfundamentos das capacidades dinâmicas. Foram aplicados testes não-paramétricos em empresas portuguesas, totalizando 137 respondentes com questionários válidos (somente gestores de negócio e TI). Em resposta ficou demonstrado que as 3 tecnologias, IoT, Big Data e IA, em proporções diferentes de desempenho, são utilizadas por essas organizações nos 3 microfundamentos (analisar o ambiente, aproveitar oportunidades e gerenciar ameaças e transformações) das capacidades dinâmicas.

A pesquisa Hsu, Tsaih, \& Yen (2018) explora o papel da evolução dos departamentos de TI em organizações não orientadas para as TIC. O objeto em estudo foi o NPM um dos principais museus do mundo, localizado em Taiwan, o qual está na vanguarda do conteúdo criativo gerado por TI e do inovador desenvolvimento de serviços com tecnologia TIC, expandindo o tradicional espaço on-line do museu para atender aos desafios atuais. Foram realizadas entrevistas profissionais do NPM de vários departamentos e especialistas externos (formuladores de políticas, acadêmicos e ex-funcionários do NPM) O artigo tomou como base 
a obra de Augier \& Teece (2006) para ilustrar os microfundamentos do processo de transformação que podem ser uma fonte de vantagem sustentável se desenvolvido por meio de habilidades difíceis de serem imitadas, que estão profundamente enraizadas na organização, como é o caso do NPM. Aqui os autores destacam as capacidades dinâmicas em que:

a) Sensing: detecta o ambiente interno, $P \& D$ e a restruturação organizacional;

b) Seizing: modelo de negócio, decisão, cultura de incentivo; estrutura organizacional;

c) Transforming: liderança, marca, gestão do conhecimento, reestrutura $\mathrm{O}$ estudo de

Liu, Chen, \& Chou (2011) explora o processo de desenvolvimento da transformação digital por meio de um projeto de banco eletrônico. O objeto de estudo foi o CBC Bank privatizado em 1971. Foram realizadas 17 entrevistas em profundidade e dados secundários foram coletadas e analisadas. O estudo explorou oito fatores críticos necessários para a implementação bem-sucedida do projeto de e-banking, sendo eles: (i) caminho histórico, (ii) confiança incorporada, (iii) dispositivo de ligação dedicado, (iv) equipe altamente autorizada e (v) colaboração, personalização, integração de TI e agilidade de reconfiguração. Os resultados demonstram que o gerenciamento da transformação digital pode ser desafiador, mas a conscientização e a preparação para sua implementação exigem análises de recursos, capacidade dinâmicas e demandas externas por intermédio da perspectiva de ajuste de recursos.

\subsection{SÍNTESE DOS RESULTADOS}

A análise dos artigos concentrou-se em identificar como as capacidades dinâmicas podem apoiar as organizações no processo de transformação digital. Os principais elementos encontrados foram: a) esforço pela renovação estratégica contínua e em modelos de negócios; b) melhoria na maturidade da força de trabalho; c) na construção de novas capacidades de governança digital; d) capacidade da empresa de integrar, construir e configurar recursos internos e competências externas para lidar com mudanças rápidas do ambiente; e) adaptação aos ecossistemas de negócios e moldá-los através de inovação e da colaboração com outras instituições e moldar ameaças e aproveitar oportunidades; f) capacidade de manter a competitividade; g) absorção do impacto das inovações digitais; h) busca por resultados em processos envolvendo análise de ambiente em recursos organizacionais, vinculados a gestão de 
riscos; i) disseminação interna do conhecimento e inovação; j) $\mathrm{Na}$ capacidade de (sensoriamento) de organizações não orientadas para TICs em termos de P\&D gerado por TI e / ou serviços habilitados por TIC; k) através das capacidades (transformadoras) associadas à liderança ao branding.

Balizados por estes estudos, é possível concluir que as capacidades dinâmicas podem auxiliar as organizações no processo de transformação digital detectando oportunidades e ameaças, com o intuito e adaptar-se a estas, gerando vantagem competitiva organizacional.

\section{CONSIDERAÇÕES FINAIS}

Este artigo se propôs a realizar uma revisão integrativa nas bases de dados Scopus e WOS, nos últimos dez anos, procurando responder a seguinte questão de pesquisa: Como as capacidades dinâmicas podem apoiar as organizações no processo de transformação digital.

Foram colecionados 14 artigos dos quais 7 deles fizeram parte da análise de dados desta pesquisa onde apareceram 2 casos da China, 2 casos de Taiwan e 1 caso de cada, da Argentina, Brasil Portugal e Alemanha.

A revisão da literatura apresentou conceitos acerca da transformação digital, fenômeno e que está voltado para as mudanças que as tecnologias digitais podem trazer no modelo de negócios de uma empresa, que resultam em produtos alterados ou estruturas organizacionais ou na automação de processos (HESS, BENLIAN \& WIESBÖCK 2016).

As capacidades dinâmicas são as capacidades que a empresas tem para integrar, construir e configurar recursos internos e competências externas para lidar com mudanças rápidas do ambiente (TEECE, 2018).

Os principais resultados apontam que as capacidades dinâmicas podem apoiar as organizações em processo de transformação digital uma vez que as permitem adaptar-se aos ecossistemas de negócios, a moldá-los por intermédio da inovação e da colaboração com outras instituições e na própria transformação organizacional. 


\section{REFERÊNCIAS}

Analia Sanchez, M. (2017). A Framework to Assess Organizational Readiness for the Digital Transformation. Dimension Empresarial, 15(2), 27-40.

Augier, M., \& Teece, D. J. (2006). Understanding complex organization: the role of know-how, internal structure, and human behavior in the evolution of capabilities. Industrial and Corporate Change, 15(2), 395-416.

Barney, J. (1991). Firm resources and sustained competitive advantage. Journal of Management, 17(1), 99-120.

Bhamra, R., Dani, S., \& Burnard, K. (2011). Resilience: the concept, a literature review and future directions. International Journal of Production Research, 49(18), 5375-5393.

Brynjolfsson, E., \& Hitt, L. M. (2000). Beyond computation: Information technology, organizational transformation and business performance. Journal of Economic Perspectives, 14(4), 23-48.

Carcary, M., Doherty, E., \& Conway, G. (2016). A dynamic capability approach to Digital transformation: A focus on key foundational themes. In S. P. Quaresma R. Guerreiro A. (Ed.), Proceedings of the European Conference on IS Management and Evaluation, ECIME (pp. 20-28). Academic Conferences and Publishing International Limited. Retrieved

Dabab, M., \& Weber, C. (2018). Business Intelligence and Data Analytics as a Driver of Dynamic Capability Strategic Approach. In 2018 Portland International Conference on Management of Engineering and Technology (PICMET) (pp. 1-9). IEEE.

De Mendonca, C M C, \& De Andrade, A. M. V. (2018a). Microfoundations of dynamic capabilities and their relations with elements of digital transformation in Portugal. In L.T. A. G. R. Rocha A. Cota M.P. (Ed.), Iberian Conference on Information Systems and Technologies, CISTI (Vol. 2018-June, pp. 1-6). IEEE Computer Society. https://doi.org/10.23919/CISTI.2018.8399414

De Mendonca, C M C, \& De Andrade, A. M. V. (2018b). Use of the elements of digital transformation in dynamic capabilities in a Brazilian capital [Uso de Elementos da Transformação Digital nas Capacidades Dinămicas em uma Capital Brasileira]. In L.-T. A. G. R. Rocha A. Cota M.P. (Ed.), Iberian Conference on Information Systems and Technologies, CISTI (Vol. 2018-June, pp. 1-6). IEEE Computer Society. https://doi.org/10.23919/CISTI.2018.8399369

De Mendonca, Claudio Marcio Campos, \& de Andrade, A. M. V. (2018). Microfoundations of dynamic capabilities and their relations with elements of digital transformation in Portugal. In 2018 13th Iberian Conference on Information Systems and Technologies (CISTI) (pp. 1-6). IEEE. https://doi.org/10.23919/CISTI.2018.8399414 
Dugstad, J., Eide, T., Nilsen, E. R., \& Eide, H. (2019). Towards successful digital transformation through co-creation: A longitudinal study of a four-year implementation of digital monitoring technology in residential care for persons with dementia. BMC Health Services Research, 19(1). https://doi.org/10.1186/s12913-019-4191-1

Fitzgerald, M., Kruschwitz, N., Bonnet, D., \& Welch, M. (2014). Embracing digital technology: A new strategic imperative. MIT Sloan Management Review, 55(2), 1.

Helfat, C. E., \& Winter, S. G. (2011). Untangling dynamic and operational capabilities: Strategy for the (N) ever-changing world. Strategic Management Journal, 32(11), 1243-1250.

Hess, T., Matt, C., Benlian, A., \& Wiesböck, F. (2016). Options for formulating a digital transformation strategy. MIS Quarterly Executive, 15(2).

Hsu, C.-C., Tsaih, R.-H., \& Yen, D. C. (2018). The Evolving Role of IT Departments in Digital Transformation. Sustainability, 10(10). https://doi.org/10.3390/su10103706

Karimi, J., \& Walter, Z. (2015). The Role of Dynamic Capabilities in Responding to Digital Disruption: AFactor-Based Study of the Newspaper Industry. Journal Of Management Information Systems, 32(1), 39-81. https://doi.org/10.1080/07421222.2015.1029380

Konlechner, S., Müller, B., \& Güttel, W. H. (2018). A dynamic capabilities perspective on managing technological change: a review, framework and research agenda. International $\begin{array}{lllll}\text { Journal of Technology } & \text { Management, } & 76(3 / 4), & \end{array}$ https://doi.org/10.1504/IJTM.2018.091285

Korhonen, J. J., \& Halen, M. (2017). Enterprise Architecture for Digital Transformation. In 2017 IEEE 19th Conference on Business Informatics (CBI) (pp. 349-358). IEEE. https://doi.org/10.1109/CBI.2017.45

Liu, D.-Y., Chen, S.-W., \& Chou, T.-C. (2011a). Resource fit in digital transformation Lessons learned from the CBC Bankglobal e-banking project. MANAGEMENT DECISION, 49(910), 1728-1742. https://doi.org/10.1108/00251741111183852

Liu, D.-Y., Chen, S.-W., \& Chou, T.-C. (2011b). Resource fit in digital transformation Lessons learned from the CBC Bank global e-banking project. MANAGEMENT DECISION, 49(910), 1728-1742. https://doi.org/10.1108/00251741111183852

Porter, M. E., \& Heppelmann, J. E. (2014). How smart, connected products are transforming competition. Harvard Business Review, 92(11), 64-88.

Riera, C., \& Iijima, J. (2019a). The Role of IT and Organizational Capabilities on Digital Business Value. PACIFIC ASIA JOURNAL OF THE Association For Information Systems, 11(2), 67-95. https://doi.org/10.17705/1pais.11204

Riera, C., \& Iijima, J. (2019b). The Role of IT and Organizational Capabilities on Digital Business Value. Pacific Asia Journal of the Association For Information Systems, 11(2), 
Rogers, D. L. (2016). The digital transformation playbook: Rethink your business for the digital age. Columbia University Press.

Sánchez, Marisa A, \& Zuntini, J. I. (2018). Organizational readiness for the digital transformation: a case study research. Revista Gestão \& Tecnologia, 18(2), 70-99. https://doi.org/10.20397/2177-6652/2018.v18i2.1316

Sánchez, Marisa Analía. (2017). A framework to assess organizational readiness for the digital transformation. Dimensión Empresarial, 15(2), 27-40.

Santos, N. dos. (2000). Gestão estratégica do conhecimento. Material Do Curso de PósGraduação Em Engenharia de Produção-UFSC/Florianópolis.

Surmeier, A. (2020). Dynamic capability building and social upgrading in tourism - Potentials and limits of sustainability standards. Journal of Sustainable Tourism, 28(10), 1498-1518. https://doi.org/10.1080/09669582.2020.1744615

Teece, D.; Pisano, G.; Shuen, A. (1997). Dynamic capabilities and strategic management. Strategic Management Journal, 18.

Teece, D.; Pisano, G. (1994). The dynamic capabilities of firms: an introduction. Industrial and Corporate Change, 3.

Teece, D. J. (2007). Explicating dynamic capabilities: the nature and microfoundations of (sustainable) enterprise performance. Strategic Management Journal, 28(13), 1319-1350.

Teece, D. J. (2016). Dynamic capabilities and entrepreneurial management in large organizations: Toward a theory of the (entrepreneurial) firm. European Economic Review, 86, 202-216. https://doi.org/10.1016/j.euroecorev.2015.11.006

Teece, D. J. (2018). Business models and dynamic capabilities. Long Range Planning, 51(1), 40-49. https://doi.org/10.1016/j.lrp.2017.06.007

Teece, D. J., Pisano, G., \& Shuen, A. (1997). Dynamic capabilities and strategic management. Strategic Management Journal, 18(7), 509-533.

Torres-Carrión, P. V., González-González, C. S., Aciar, S., \& Rodríguez-Morales, G. (2018). Methodology for systematic literature review applied to engineering and education. In 2018 IEEE Global Engineering Education Conference (EDUCON) (pp. 1364-1373). IEEE.

Vial, G. (2019a). Understanding digital transformation: A review and a research agenda. The Journal of Strategic Information Systems. https://doi.org/10.1016/j.jsis.2019.01.003

Vial, G. (2019b, February). Understanding digital transformation: A review and a research 

agenda.
Journal
of
Strategic
Information
Systems.
https://doi.org/10.1016/j.jsis.2019.01.003

Warner, K. S. R., \& Wäger, M. (2019). Building dynamic capabilities for digital transformation: An ongoing process of strategic renewal. Long Range Planning, 52(3), 326-349. https://doi.org/10.1016/j.lrp.2018.12.001 